\title{
Dopamine agonist withdrawal in hyperprolactinemia: when and how
}

\author{
Roberto Salvatori $\mathbb{1}^{1}$
}

Received: 9 October 2017 / Accepted: 3 November 2017 / Published online: 9 November 2017

(C) Springer Science+Business Media, LLC, part of Springer Nature 2017

Dopaminergic agents (DA) have been commonly used to treat hyperprolactinemia since the late 1970's. Since the mid 1990 's, after a seminal study that showed it to be more effective and better tolerated than bromocriptine [1], cabergoline has become for most endocrinologists the drug of choice to treat various forms of hyperprolactinemia. However, more recently worries about cabergoline effects on the heart valve anatomy and function were raised by two studies that looked at Parkinson disease patients on high dosages of this drug $[2,3]$. These effects are thought to be mediated by cabergoline action on the serotonin (5-hydroxytryptamine, or 5-HT) receptor $2 \mathrm{~B}$. These findings caused an expert writing the editorial that accompanied these two papers to advocate that "practitioners should avoid prescribing drugs that are potent 5-HT2B-receptor agonists-a growing list of medications that now includes (...) cabergoline" [4]. However, in the Schade study [2], a significant risk of valvular disease was observed only in patients taking more than $3 \mathrm{mg}$ per day of cabergoline, a dosage almost never used in the treatment of hyperprolactinemia. Similarly, in the Zanettini paper, the average daily dose of cabergoline was $3.6 \mathrm{mg}$ [3]. Indeed, recent literature seems to be reassuring about the lack of obvious risk of heart valve damage in patients treated with cabergoline at the dosages more commonly used for hyperprolactinemia (generally below $3 \mathrm{mg}$ per week) [5]. Nevertheless, a small degree of worry remains among practitioners about the long-term

Roberto Salvatori

salvator@jhmi.edu

1 Division of Endocrinology and Pituitary Center, Johns Hopkins School of Medicine, 1830 E. Monument st. \#333, Baltimore, MD 21287, USA effects of low dosages of cabergoline. In this scenario, the 2003 paper from Colao et al. that showed that a significant percentage $(66.5 \%)$ of patients who had received cabergoline for longer than 2 years for non-tumoral hyperprolactinemia, micro-prolactinomas and macro-prolactinomas maintained a normal prolactin level after cabergoline withdrawal became even more relevant [6]. Of notice, this was a selected group of patients (200), who had normal prolactin on a minimal dose of cabergoline, and whose magnetic resonance imaging (MRI) showed no tumor or tumor reduction of $50 \%$ or more. No information was provided on what percentage of their hyperprolactinemia patient population these subjects represented. In a follow-up paper, the same group identified a serum prolactin level $\leq 5.4 \mathrm{ng} / \mathrm{mL}$ and maximal residual tumor diameter $\leq 3.1 \mathrm{~mm}$ on MRI as predictors of remission [7]. These observations prompted both the Pituitary and the Endocrine Society to recommend a trial of DA withdrawal in selected hyperprolactinemic patients $[8,9]$. Follow-up studies by other groups have reported a lower rate of successful DA withdrawal than reported in the Colao paper. A paper published in this issue of Endocrine by Xia et al. [10] reports the results of a meta-analysis of all available DA withdrawal trials, looking at a total of 1106 patients form 24 studies. They found an overall success rate of cabergoline withdrawal of $36.6 \%$, lower than in the original Colao paper, but higher than what reported $(21 \%)$ in a previous more limited meta-analysis [11]. They report that a low-dose maintenance therapy with cabergoline ( $0.5 \mathrm{mg}$ per week), and a significant reduction in tumor size were independent factors affecting the success rate of DA withdrawal. They also report higher success rate in patients using cabergoline as only treatment than in patients treated with bromocriptine, and in patients treated for longer than 2 years. Interestingly, 
contrarily to the previous meta-analysis that found that patients with idiopathic hyperprolactinemia were more likely to remain in remission after DA withdrawal compared with microprolactinomas and macroprolactinomas patients, Xia et al. found no difference in the three clinical scenarios. Their data showed that more than half of patients on low dose cabergoline ( $\leq 0.5 \mathrm{mg}$ per week) can safely stop the drug, and recommend withdrawal in patients who have been on cabergoline for at least 2 years, whose prolactin has been low $(\leq 10 \mathrm{ng} / \mathrm{mL})$ for one year, and have achieved significant tumor size reduction. Although not specifically reported in this meta-analysis, a further reassuring factor that should convince clinicians to make at least one attempt to stop cabergoline is that, when withdrawal is not successful, serum prolactin increase occurs before any visible increase in tumor size by MRI $[6,12]$.

The results of the Xia meta-analysis certainly help clinician deciding which DA to choose (cabergoline is more likely to cause permanent remission than bromocriptine) and understand in which biochemical and radiological scenario DA withdrawal is advisable. However, several questions about medical management of hyperprolactinemia remain unanswered. One is a question that often patients ask when therapy is started: "is this a lifetime treatment"? Regrettably, at present, no data are available to help the clinician answering such question before therapy is started. We do not know what percentage of hyperprolactinemic patients reaches the conditions associated with good chance of successful DA withdrawal, nor do we have good predictors of responsiveness to DA therapy. While in the Xia meta-analysis a serum prolactin $>200 \mathrm{ng} / \mathrm{mL}$ at diagnosis was associated with slightly higher chance of DA withdrawal success (36.5 vs. $28.8 \%$ ) this only gives limited information to the patient. In my personal experience with more than 300 prolactinoma patients, I note that the degree of responsiveness to DA therapy generally becomes obvious during the first few months of therapy, and patients whose prolactin level drops rapidly are more likely to fit the withdrawal criteria two years or longer into therapy. A second important question that remains unanswered is how low the clinician should push prolactin level during DA therapy. Is it enough to normalize prolactin or should DA dose be increased in order to bring prolactin as low as possible during the initial phase of therapy? To my knowledge, no clinical trial or retrospective study has looked at this question. A third unanswered question is whether there is a length of therapy required to normalize prolactin beyond which the chance of successful DA withdrawal becomes a futile exercise. Finally, what is the success rate of a second DA withdrawal attempt after an unsuccessful one? Two small studies looked at this [13, 14]. In both trials patients who had failed a first cabergoline withdrawal were treated for at least additional 2 years before a second withdrawal was attempted. Out of a total of 51 patients, $35(69 \%)$ recurred. While this justifies a second cabergoline withdrawal attempt, the numbers are small, and larger studies are needed.

In conclusion, while we now know more about which hyperprolactinemic patients are more likely to be successfully withdrawn from DA therapy, several questions about medical treatment of hyperprolactinemia remain unanswered. Hopefully the answers will come from additional studies. In the meantime, DA withdrawal should be considered in all patients who meet the criteria described in the Xia meta-analysis, with careful monitoring of serum prolactin.

\section{Compliance with ethical standards}

Conflict of interest The author declares that he has no competing interests.

Ethical approval This article does not contain any studies with human participants or animals performed by the author.

\section{References}

1. J. Webster, G. Piscitelli, A. Polli, C.I. Ferrari, I. Ismail, M.F. Scanlon, A comparison of cabergoline and bromocriptine in the treatment of hyperprolactinemic amenorrhea-Cabergoline Comparative Study Group. N. Engl. J. Med. 331, 904-909 (1994)

2. R. Schade, F. Andersohn, S. Suissa, W. Haverkamp, E. Garbe, Dopamine agonists and the risk of cardiac-valve regurgitation. N. Engl. J. Med. 356, 29-38 (2007)

3. R. Zanettini, A. Antonini, G. Gatto, R. Gentile, S. Tesei, G. Pezzoli, Valvular heart disease and the use of dopamine agonists for Parkinson's disease. N. Engl. J. Med. 356, 39-46 (2007)

4. B.L. Roth, Drugs and valvular heart disease. N. Engl. J. Med. 356, 6-9 (2007)

5. R.S. Auriemma, R. Pivonello, L. Ferreri, P. Priscitelli, A. Colao, Cabergoline use for pituitary tumors and valvular disorders. Endocrinol. Metab. Clin. North. Am. 44, 89-97 (2015)

6. A. Colao, A. Di Sarno, P. Cappabianca, C. Di Somma, R. Pivonello, G. Lombardi, Withdrawal of long-term cabergoline therapy for tumoral and nontumoral hyperprolactinemia. N. Engl. J. Med. 349, 2023-2033 (2003)

7. A. Colao, A. Di Sarno, E. Guerra, R. Pivonello, P. Cappabianca, F. Caranci, A. Elefante, L.M. Cavallo, F. Briganti, S. Cirillo, G. Lombardi, Predictors of remission of hyperprolactinaemia after long-term withdrawal of cabergoline therapy. Clin. Endocrinol. (Oxf). 67, 426-433 (2007)

8. F.F. Casanueva, M.E. Molitch, J.A. Schlechte, R. Abs, V. Bonert, M.D. Bronstein, T. Brue, P. Cappabianca, A. Colao, R. Fahlbusch, H. Fideleff, M. Hadani, P. Kelly, D. Kleinberg, E. Laws, J. Marek, M. Scanlon, L.G. Sobrinho, J.A. Wass, A. Giustina, Guidelines of the Pituitary Society for the diagnosis and management of prolactinomas. Clin. Endocrinol. (Oxf). 65, 265-273 (2006)

9. S. Melmed, F.F. Casanueva, A.R. Hoffman, D.L. Kleinberg, V.M. Montori, J.A. Schlechte, J.A. Wass, Endocrine Society. Diagnosis and treatment of hyperprolactinemia: an Endocrine Society clinical practice guideline. J. Clin. Endocrinol. Metab. 96, 273-288 (2011) 
10. M.Y. Xia, X.H. Lou, S.J. Lin, Z.B. Wu, Optimal timing of dopamine agonist withdrawal in patients with hyperprolactinemia: a systematic review and meta-analysis. Endocrine (2017). https:// doi.org/10.1007/s12020-017-1444-9

11. O.M. Dekkers, J. Lagro, P. Burman, J.O. Jørgensen, J.A. Romijn, A.M. Pereira, Recurrence of hyperprolactinemia after withdrawal of dopamine agonists: systematic review and meta-analysis. J. Clin. Endocrinol. Metab. 95, 43-51 (2010)

12. J. Kharlip, R. Salvatori, G. Yenokyan, G.S. Wand, Recurrence of hyperprolactinemia after withdrawal of long-term cabergoline therapy. J. Clin. Endocrinol. Metab. 294, 2428-2436 (2009)
13. R. Kwancharoen, R.S. Auriemma, G. Yenokyan, G.S. Wand, A. Colao, R. Salvatori, Second attempt to withdraw cabergoline in prolactinomas: a pilot study. Pituitary. 17, 451-456 (2014)

14. L. Vilar, J.L. Albuquerque, P.S. Gadelha, F. Rangel Filho, A.M. Siqueira, M.M. da Fonseca, K.F. Viana, B.S. Gomes, R. Lyra, Second attempt of cabergoline withdrawal in patients with prolactinomas after a failed first attempt: is it worthwhile? Front. Endocrinol. (Lausanne) 6, 11 (2015) 